Observations have been made with this system nearly every day for more than eighteen months, although records representing about 15 per cent of the total observing time have had to be rejected from the present analysis owing to the occurrence of severe 'scintillation' caused by diffraction in ionospheric irregularities ${ }^{2}$. It was found that, of approximately a hundred radio stars examined, none showed any variation of intensity greater than 10 per cent; a more detailed analysis of some of the more intense ones suggested that their intensity was, in fact, constant to better than 5 per cent; but it is difficult, at this wave-length, to eliminate the effects of residual ionospheric irregularities for variations smaller than this.

It is therefore concluded that none of the radio stars observed varies by more than 0.1 magnitude with any period shorter than about 1,000 days; observations of the more intense stars suggest that their intensities may, in fact, be constant to within 0.05 magnitude.

This result is of interest not only for comparison with the behaviour of visible stars, but also in contrast to the marked day-to-day variations in the intensity of the metre-wave radiation from the sun.

This work forms part of a programme of research which is supported at the Cavendish Laboratory by the Department of Scientific and Industrial Research.

M. RYLE

B. ELSMORE

Cavendish Laboratory, Cambridge.

Aug. 8.

${ }^{2}$ Ryle, M., Smith, F. G., and Elsmore, B., Mm. Not. Roy. Astro. Soc., 110

${ }^{2}$ Ryle, M., and Hewish, A., Mon. Not. Roy. Astro. Soc., 110, 381 $(1950)$.

\section{Nuclear Spin of Rubidium-86}

Tне nuclear spin $I$ of rubidium-86 has been measured by the atomic beam method using magnetic resonance; a mass spectrometer was employed to select the correct isotope ${ }^{1}$. A pronounced resonance was obtained at a frequency of $2.36 \mathrm{Mc} / \mathrm{sec}$. in a magnetic field of $4 \cdot 22$ gauss, which gives $I=2$. This value is in agreement with the observed shape of the $\beta$-spectrum ${ }^{2}$. The hyperfine structure separation was found to be $3,900 \pm 100 \mathrm{Mc}$./sec., which means a magnetic moment of $1 \cdot 66 \pm 0 \cdot 4$ nuclear magnetons. The results suggest that the magnetic moment is probably negative and it would then have a value of $-1.68+0.01$ nuclear magnetons. It is planned to extend the measurements to obtain a more accurate value of the magnetic moment and definitely establish its sign.

The rubidium- 86 was prepared by the ${ }^{88} \mathrm{Sr}(d, \alpha){ }^{88} \mathrm{Rb}$ reaction. I wish to thank the Physics Department of the University of Birmingham for carrying out this bombardment, and Dr. A. G. Maddock, of the Radiochemistry Laboratory, Cambridge, for the chemical separation of the rubidium- 86 .

Cavendish Laboratory,

\section{E. H. BELLAMY} Cambridge. Aug. 16.

1 Davis, Nagle and Zacharias, Phys. Rev., 76, 1068 (1949). ¿ Zaffarano, Kern and Mitchell, Phys. Rev., 74, 682 (1948)

\section{Scattering of 146-MeV. Protons by Deuterons}

THE proton beam and the counting apparatus described in an earlier communication ${ }^{1}$ have now been used for a study of elastic proton-deuteron scattering. The results are shown in the accompanying graph.

The scatterer was a rectangular strip of heavy water contained in a capsule made of thin aluminium sheet. Elastic proton-deuteron collisions were observed by detecting in coincidence both the scattered protons and the corresponding recoil deuterons. When the angle of scattering was less than $67^{\circ}$ or greater than $85^{\circ}$, the geometry of the arrangement left no doubt as to which counter was detecting the protons in these events. Between these two angles it was not practicable to determine the cross-section except at $76^{\circ}$, where the recoil angles of the two particles are actually equal.

Various background effects were subtracted with the help of a second scatterer filled with normal water. Proton-proton collisions in the hydrogen content of the normal water were not counted, again because of the geometry of the arrangement. All background effects should have been unchanged by the substitution of normal for heavy water, with the exception of that due to inelastic proton-deuteron scattering followed by the detection of both protons. There are three final particles involved in this process, so that the recoil angles of the two protons are not uniquely connected. A consideration of the solid angle subtended by the counters suggested that this source of background could be neglected, and this conclusion was supported by an experiment in which the geometry of the detectors was varied.

The uncertainties shown in the graph are the statistical errors in the determination of the relative values at different angles; it is considered that systematic errors were small in comparison with these. The absolute cross-section was fixed by observing proton-proton scattering with the same apparatus, the cross-section for that process being taken ${ }^{1}$ as $4.99 \pm 0.31$ millibarns. The absolute calibration of the proton-deuteron experiment is subject to an overall standard deviation of $7 \cdot 8$ per cent.

It is hoped to improve the accuracy of these results and later to obtain some information about neutron-deuteron scattering at the same energy. A

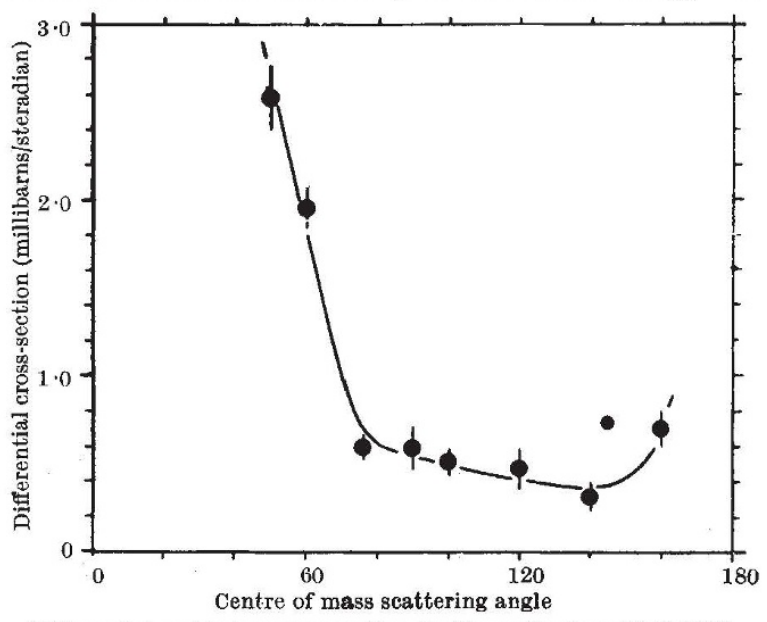

Differential scattering cross-section for the scattering of $146-\mathrm{MeV}$. protons by deuterons, as a function of angle in the centre of mass system 